\title{
GENERACIÓN MILLENNIAL: CONSUMO GASTRONÓMICO Y TURÍSTICO
}

MILLENNIAL GENERATION: GASTRONOMIC AND TOURIST CONSUMPTION

Ilse Alexandra Quevedo Pérez*, Enriqueta Pérez Zurita**

*Doctora en Educación. Profesora Investigadora. Universidad Juárez Autónoma de Tabasco.

**Maestra en Ciencias de la Educación. Profesora Investigadora. Universidad Juárez Autónoma de Tabasco.

Dirección para recibir correspondencia: I.a.ilsequevedo@gmail.com 
GENERACIÓN MILLENNIAL: CONSUMO GASTRONÓMICO Y TURÍSTICO

\section{RESUMEN}

OBJETIVO: Conocer los gustos y preferencias de la Generación Millennial con respecto a sus requerimientos de consumo de alimentos y servicios turísticos.

MATERIAL Y MÉTODO: El enfoque de la investigación fue cualitativo, utilizando las técnicas de recolección en campo a través de un sondeo flash digital, donde se utilizó un instrumento de diseño propio. Asimismo, se desarrolló trabajo de gabinete con revisión documental. El informante clave fue un grupo de personas pertenecientes a la Generación Millennial.

RESULTADOS: Se encontró que los Millennials son una generación que se caracteriza por el uso de los dispositivos electrónicos para reservar anticipadamente sus viajes, que suelen ser frecuentes. En cuanto a sus gustos y frecuencia de consumo gastronómico, prefieren comida personalizada mayormente.

CONCLUSIONES: Los principales hallazgos de la investigación muestran una congruencia entre la teoría y las declaraciones de los informantes clave, respecto a los gustos y preferencias en la realización de actividades y requerimientos de servicios de gastronomía y turismo, por parte de la Generación Millennial.

PALABRAS CLAVE: Generación Millennial. Alimentos. Gastronomía. Turismo.

\section{ABSTRACT}

OBJECTIVE: To know the tastes and preferences of the Millennial Generation with respect to their food consumption requirements and tourist services.

MATERIAL Y METHOD: The focus of the research was qualitative, using field collection techniques through a digital flash probing, where a self-designed instrument was used. Also, cabinet work was developed with a documentary review. The key informant was a group of people belonging to the Millennial Generation.

RESULTS: It was found that Millennials are a generation that is characterized by the use of electronic devices to book their trips in advance, which are often frequent. As for their tastes and frequency of gastronomic consumption, they mostly prefer personalized food. 
GENERACIÓN MILLENNIAL: CONSUMO GASTRONÓMICO Y TURÍSTICO

CONCLUSIONS: The main findings of the research show a congruence between the theory and the declarations of the key informants, regarding the tastes and preferences in the realization of activities and requirements of gastronomy and tourist services, by the Millennial Generation

Key words: Millennial Generation. Food. Gastronomy. Tourism.

\section{INTRODUCCIÓN}

La Generación Millennial o también Ilamada "Generación Y", resulta ser un segmento muy atractivo para las empresas, ya que representa unas de las principales oportunidades de crecimiento en cuanto a la participación de mercado, puesto que son económicamente activos, por esta razón las empresas están interesadas en investigar e identificar cuáles son los gustos y preferencias, capacidad de compra, en conclusión el estilo de vida que predomina en ellos; con la finalidad de poder diseñar las mejores estrategias de mercadotecnia, para generar lealtad y satisfacción en dicho consumidor, en cuestión de investigación de mercado y desarrollo de planes estratégicos de mercadotecnia, con el propósito de llegar a ser una de las mejores opciones en el mercado de cualquier sector productivo y en especial aquel que ofrece servicios de gastronomía y turismo.

Resulta ser motivo de gran preocupación para las empresas el desconocer las tendencias de mercado, especialmente la de los Millennials, ya que este grupo de personas representará el $75 \%$ de la fuerza laboral a nivel global para el 2025 según la publicación en el resumen ejecutivo "Big demands and high expectations" The Deloitte Millennial Survey (2014). Lo anterior, confirma la importancia y trascendencia de permanecer a la vanguardia para satisfacer esta generación que posee gustos y preferencias únicas y variantes con alto potencial de consumo e influencia en el mercado.

La satisfacción de los consumidores y clientes, es fundamental para el buen funcionamiento de las empresas, por lo cual la técnica de mercadeo; es decir, mercadotecnia o marketing lleva a cabo una serie de acciones encaminadas a la creación, promoción, distribución y venta de productos tangibles e intangibles en tiempos deseados y lugares claves para el cliente (Münch, Sandoval, Torres y Ricalde, 2015). 
GENERACIÓN MILLENNIAL: CONSUMO GASTRONÓMICO Y TURÍSTICO

\section{MARCO TEÓRICO}

\section{¿Quiénes son los Millennials?}

Es importante conocer, que la Generación Millennial está agrupada en diferentes períodos que van a partir de los años 80's hasta mediados de los años 90's, esto de acuerdo a diferentes empresas de consultoría y agencias de investigación, tales como: el grupo consultor PricewaterhouseCoopers junto con la Universidad de Southern California y London Business School (2013) establecieron que el rango es a partir de 1980 a 1995; Mark McCrindle (2014) afirma que estas personas nacieron entre 1980 y 1994, Synchrony Financial (2014) afirma que va desde 1976 a 1992.

Con respecto a la conceptualización de la Generación Millennial o "Generación Y" Schiffman y Lazar (2010) afirman que también es llamada "echo boomers" y establecen una clasificación de tres segmentos: adultos con un rango de edad de 19 a 28 años, los adolescentes de 13 a 18 años de edad y preadolescentes de 8 a 12 años de edad.

Destacan que aunque existen diferencias evidentes en los rangos de edades, pertenecen a una misma generación mayormente conocida como Generación Millennial. También mencionan que, existe un grupo de personas con rango de edad de 21 a 29 años pertenecientes a las generaciones $X$ y $Y$, de los cuáles muchos ya egresaron de las universidades y tienen empleos dignos y bien remunerados, pero que todavía continúan viviendo con sus padres, también están considerando tomar la decisión de casarse después de cumplir los 30 años; esta generación reconoce que gastan más que la mayoría de la gente en comer fuera de casa, comprar ropa, diversiones y equipos electrónicos que están de moda. Sin embargo, es sabido que aún no tienen la capacidad económica para adquirir un bien inmueble.

De acuerdo con Gutiérrez-Rubí (2014), hay seis características que destacan a los Millennials en comparación con otras generaciones, las cuales son: nativos digitales, ya que son expertos en el manejo de la tecnología y prefieren el uso del internet, presentan una conducta multitareas en donde realizan diversas actividades al mismo tiempo a través del uso de diversos dispositivos digitales y medios de comunicación, actitud nomófobica ya que les resulta aterrador prescindir del uso del teléfono celular y las aplicaciones (apps), son usuarios constantes y fieles a las redes sociales las cuales son utilizadas tanto para el ámbito profesional como personal, manifiestan una actitud crítica y minuciosa ante el consumo de bienes y servicios y por último, tienen una tendencia a recibir una atención de calidad de acuerdo a sus preferencias en donde 
GENERACIÓN MILLENNIAL: CONSUMO GASTRONÓMICO Y TURÍSTICO

desean experimentar sentirse únicos y especiales al participar en la creación de productos y servicios que consume, así mismo estos los buscan de acuerdo a sus valores y creencias.

Nielsen (2015) elaboró la encuesta "Estudio Global a cerca de los estilos de vida generacionales" (The Nielsen Global Generational Lifestyles Survey) en la que participaron 30,000 personas de 60 países de manera on-line, y cuyo propósito es el conocer las diferencias en el sentir del consumidor de acuerdo a las diferentes percepciones generacionales, con respecto a los Millennials destacan las siguientes características: no es una de las principales prioridades el casarse, formar una familia, tener hijos y adquirir una casa, sin embargo todavía es importante para muchos de ellos.

En el ámbito profesional, son casi dos veces mayormente proclive a abandonar el trabajo después de un lapso de dos años si se equipara con las dos generaciones anteriores, con respecto a las actividades de ocio disfrutan la televisión e incluso el hábito de lectura.

\section{Consumo gastronómico}

Para abordar este aspecto interesante que distingue a la Generación Millennial, es conveniente conocer ¿Qué es la gastronomía? Por lo cual conforme a lo que menciona el Diccionario de la Lengua Española (2017) es "el arte de preparar una buena comida", así como el placer de degustar un buen platillo, y costumbres culinarias de un lugar en especial.

Mientras que Larousse Cocina (2017) refiere que la gastronomía de acuerdo a Charles Monselet es "la joya de todas las situaciones y de todas las edades" posteriormente en el año de 1835 la palabra gastronomía fue integrada al diccionario de la academia francesa.

El marketing gastronómico no sólo promueve recientes y originales hábitos alimenticios, sino que reconoce y armoniza con las necesidades de los consumidores en materia de desarrollo de planes estratégicos que le permitan agradar y complacer los deseos y exigencias de los mismos (Mesa editorial Merca2.0, 2015).

Nielsen (2015) reporta que los Millennials cenan fuera de casa con más frecuencia, de 10 personas seis afirman consumir alimentos fuera de casa como mínimo una vez por semana, mientras que una tercera parte tres veces por semana o más.

Por lo que respecta a los tipos de alimentos que consumen, se destacan las preferencias por la comida que les brinde beneficios a la salud, tales como: libres de gluten y cafeína, altos en 
GENERACIÓN MILLENNIAL: CONSUMO GASTRONÓMICO Y TURÍSTICO

proteínas, orgánicos, fortificados y porciones controladas, asimismo están dispuestos a pagar un precio justo en relación a la calidad, dicho sea de otra manera costo-beneficio.

Consumolab un centro AINIA (2015) realizó un estudio acerca de las tendencias en el sabor en los productos alimenticios, con el fin de conocer los gustos y preferencias de los consumidores, los Millennials tienen preferencia por el sabor dulce, pero están conscientes del consumo de comida saludable. Por lo que es importante considerar esta información para el desarrollo de nuevos productos dirigidos a este segmento de mercado.

Barragán (2015), menciona algunos aspectos que disfrutan y provocan el consumo de los Millennials, tales como: la preparación de una pizza estilo gourmet con ingredientes de calidad, comida rápida casual y saludable, rapidez en el servicio y atención, así como experiencias peculiares y únicas por supuesto, sin lugar a duda el amor por la comida orgánica. También los aspectos que les incomodan, tales como: el mal servicio al cliente, esperas prolongadas, aplicaciones móviles aburridas, no disponer del acceso al internet en los sitios donde se ofrecen y consumen alimentos.

Todas estas características presentadas en la conducta del consumidor Millennial, dejan en claro que son exigentes, con un notable sentido de urgencia, por lo que las organizaciones y empresas constantemente ofertan productos cada vez más especializados únicos y originales.

\section{Consumo turístico}

El término turismo, de acuerdo con el Diccionario de la Lengua Española (2017), significa la acción de viajar por deleite, o equipo de medios que facilitan los viajes de placer, y este procede el término en inglés tourism.

Según Kotler, Garcia de Madariaga, Flores, Bowen y Makens (2011), el sector turístico es una de las actividades productivas más importantes alrededor del mundo. En diversos países latinoamericanos, tales como: Argentina, Brasil, Chile y México, el sector turístico es uno de los principales impulsores de la economía, señalando que el servicio de alojamiento y viajes son las principales actividades que comprende el turismo.

Resulta interesante la definición presentada desde el punto de vista de Ojeda y Mármol (2016), es el área de la mercadotecnia responsable de dirigir el comercio de bienes tangibles e intangibles de aquellas empresas pertenecientes al sector del turismo. 
GENERACIÓN MILLENNIAL: CONSUMO GASTRONÓMICO Y TURÍSTICO

Una importante investigación acerca de los hábitos turísticos de los Millennials realizada por Hundredrooms y la Escuela de Negocios ESERP (2017), los Millennials Españoles acostumbran a realizar viajes por lo regular con una frecuencia de dos o cuatro veces al año mayormente, en donde planifican con tiempo y mesura las actividades a realizar durante ese período. Esta planeación oscila con un tiempo de anticipación entre menos de un mes y no más de seis meses, con el fin de tener acceso a las mejores opciones de ubicación, actividades recreativas a precios atractivos, verificando entre más de cinco alternativas para tomar la mejor decisión.

La actividad de búsqueda de hospedaje por motivos de vacaciones se realiza a través de diversos dispositivos electrónicos, en donde destaca la preferencia en el siguiente orden de importancia, computadora, teléfono celular, tablet, y agencia de viajes; mientras que la reservación del hospedaje se realiza a través de la computadora principalmente, de manera seguida el teléfono celular, las agencias de viajes y la tablet.

De acuerdo con el informe realizado por Burkhard, Kow y Fuggle (2016), a cerca de las Tendencias de Turismo 2017, Sara Burkhard afirma que la Generación Millennial es una de las más importantes en lo respecta viajes, debido a que es la más grande hoy en día, ya que ellos están dando a conocer cuáles son sus gustos de consumo de esta forma están revelando su predilección para un futuro.

También otro de los aspectos interesantes en los Millennials, es que la mayoría de ellos busca sumergirse en la cultura por medio de las degustaciones culinarias, actividades propias del lugar de destino. Asimismo, una tendencia notable es la destreza de modificar un viaje de negocios en un viaje de placer o al contrario.

\section{MATERIAL Y MÉTODO}

\section{Diseño de la investigación}

El enfoque de la investigación fue cualitativo, utilizando las técnicas de recolección en campo a través de un sondeo flash digital, donde se utilizó un instrumento de diseño propio. Asimismo, se desarrolló trabajo de gabinete con revisión documental. El informante clave fue un grupo de personas pertenecientes a la Generación Millennial. Se optó por aplicar el instrumento de forma on-line a través de redes sociales, debido a que el grupo estudiado son nativos digitales y están constantemente conectados. 
GENERACIÓN MILLENNIAL: CONSUMO GASTRONÓMICO Y TURÍSTICO

\section{Muestra}

Para fines de esta investigación cualitativa, se determinó una de muestra intencional de 61 personas de nacionalidad mexicana pertenecientes a la Generación Millennial (ver tabla 1).

Tabla 1

Edad de los encuestados

\begin{tabular}{ccc}
\hline Edades & Rango & Número de personas \\
\hline & $20-25$ años & 7 \\
& $26-30$ años & 42 \\
& $31-35$ años & 7 \\
& $36-40$ años & 5 \\
Total & & 61 \\
\hline
\end{tabular}

Fuente: Elaboración propia.

\section{Técnica de investigación}

Las técnicas de investigación fueron: sondeo flash digital, donde el instrumento fue un cuestionario con siete preguntas (ver tabla 2). Los cuestionamientos fueron referentes a actividades y preferencias turísticas y gastronómicas. Asimismo, se trabajó con la técnica de revisión documental tanto en fuentes impresas como electrónicas.

\section{Tabla 2}

Cuestionario Generación Millennial: consumo gastronómico y turístico

\begin{tabular}{cl}
\hline Sector & \multicolumn{1}{c}{ Preguntas } \\
\hline \multirow{2}{*}{ Turismo } & ¿Con qué frecuencia viaja durante el año? \\
& ¿C través de qué medios realiza la reservación? \\
& ¿Cuál es el destino que visita con mayor frecuencia? \\
\hline \multirow{2}{*}{ Gastronomía } & ¿Con que frecuencia come fuera de casa? \\
& ¿Cuál es el motivo por el cual come fuera de casa? \\
& ¿Qué tipo de comida consume?
\end{tabular}

Fuente: Elaboración propia. 


\section{RESULTADOS}

Los hallazgos obtenidos en el sondeo flash online, se presentan en dos secciones; la primera referida al sector turístico y la segunda al sector gastronómico.

\section{- Turismo}

En cuanto a frecuencia de viaje, se observa que en general lo realizan entre una o dos veces anualmente (ver figura 1).

\section{¿Con qué frecuencia viaja durante el año?}

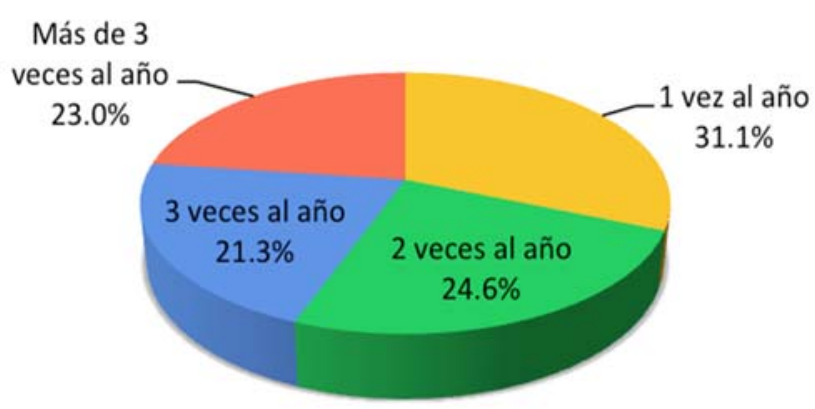

Figura 1. Frecuencia de viaje de los Millennials.

Fuente: Elaboración propia.

La razón por la que viajan es principalmente por placer (54.1\%), seguidamente de la combinación de este con el trabajo con un $41 \%$ y un grupo mínimo de $4.9 \%$ afirmó realizarlo sólo por cuestiones laborales. Sin embargo, en lo concerniente a las actividades académicas no parece ser un motivo importante por el cual viajar (ver figura 2).

\section{¿Cuál es el principal motivo de viaje?}

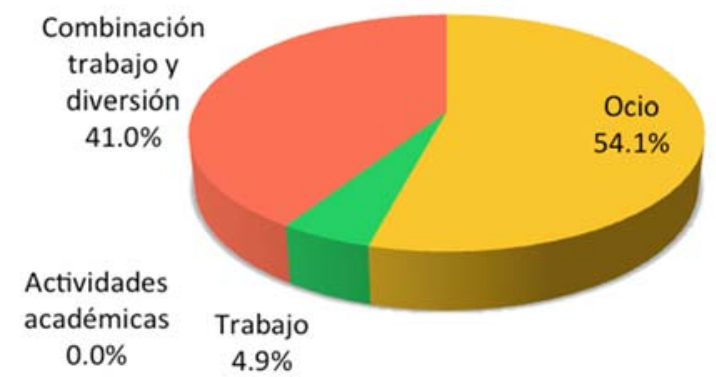

\section{Figura 2. Razón principal de viajar.}

Fuente: Elaboración propia. 
El proceso de reservación de viajes lo llevan a cabo principalmente a través de medios electrónicos, donde se destaca el uso de la computadora, con una afirmación del 63.9\%, y un 29.5\% por medio del teléfono celular; el uso de la tablet aún para los Millennials mexicanos no es aparentemente una opción, por lo que se refiere al uso del servicio de las agencias de viajes, es un porcentaje mínimo de $6.6 \%$ (ver figura 3).

\section{¿A través de qué medios realiza la reservación?}

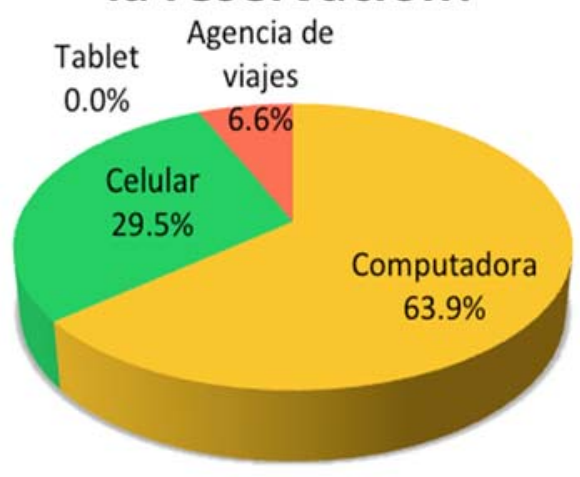

Figura 3. Medios utilizados para realizar la reservación.

Fuente: Elaboración propia.

Los principales destinos que visitan los Millennials mexicanos son preferentemente nacionales con un $78.7 \%$, seguido por los sitios locales con un 13.1\%; sin embargo, con una menor pero no menos importante tendencia es visitar lugares internacionales con un $8.2 \%$ (ver figura 4).

\section{¿Cuál es el destino que visita con mayor frecuencia?}

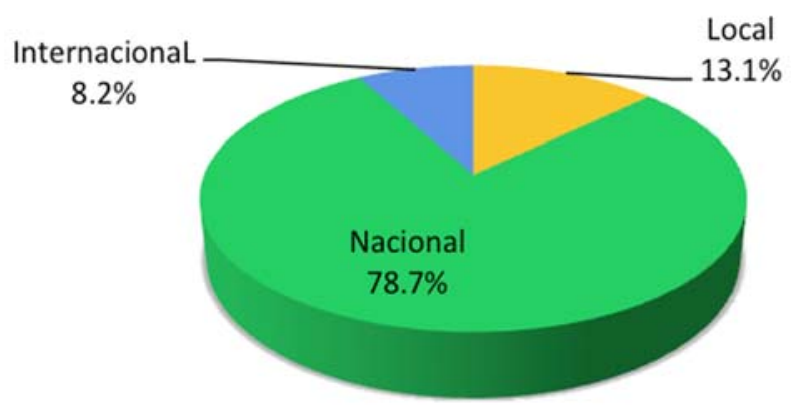

Figura 4. Destino de visita con mayor frecuencia.

Fuente: Elaboración propia. 


\section{- Gastronomía}

La frecuencia de consumo de alimentos fuera de casa, un 42.6\% manifiestan que lo realizan más de tres veces por semana, seguido de un 23\% que lo realiza dos veces por semana, mientras que comer fuera de casa para $19.7 \%$, es una vez por semana, y por último, están aquellos que comen sólo los fines de semana fuera de casa con un 14.8\% (ver figura 5).

\section{¿Con qué frecuencia come fuera de casa?}

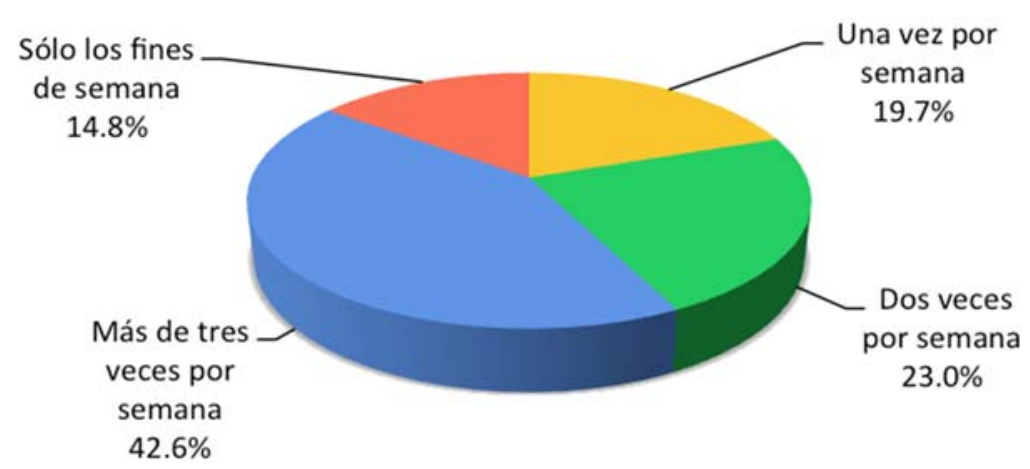

Figura 5. Frecuencia de consumo de alimentos fuera de casa por semana.

Fuente: Elaboración propia.

En lo concerniente al principal motivo por el cual se come fuera de casa, el 50.8\% afirma que es por placer, el $31.1 \%$ por cuestiones de trabajo, mientras que un $18 \%$ afirma que es por necesidad (ver figura 6).

\section{¿Cuál es el motivo por el qué come fuera de casa?}

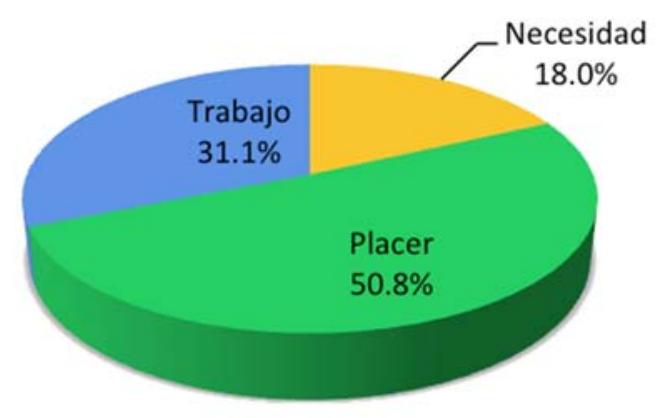

Figura 6. Motivos por el cual se come fuera de casa.

Fuente: Elaboración propia. 
De acuerdo con los diversos tipos de comida que se ofertan en el mercado el $27.9 \%$ prefiere la comida personalizada, mientras existe una tendencia marcada en cuanto a precio justo y comida rápida con el mismo porcentaje de 24.6\%, y por último, la comida saludable es la favorita de un 23\%. Este último hallazgo, se aproxima al reportado por Camacho y Camacho (2017), en relación a que las personas entre 20 y 30 años prefieren consumo de alimentos saludables en un $19.4 \%$ (ver figura 7 ).

\section{¿Qué tipo de comida consume?}

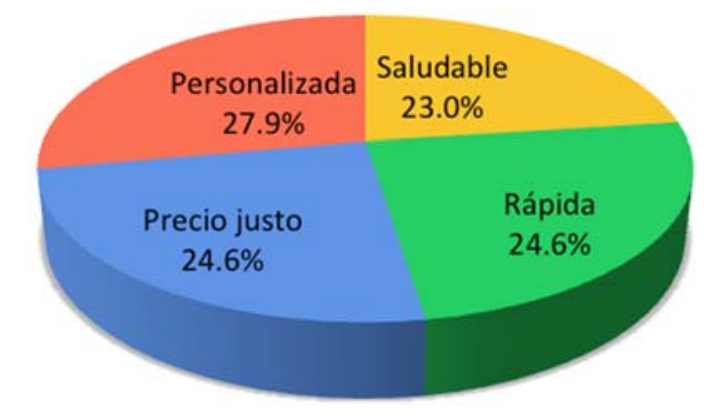

\section{Figura 7. Preferencia en los tipos de comida.}

Fuente: Elaboración propia.

\section{CONCLUSIONES}

Los resultados de la investigación, alcanzaron el objetivo de analizar los gustos y preferencias de la Generación Millennial con respecto a sus requerimientos de consumo de alimentos y servicios turísticos. Se confirma que las aportaciones en materia de comportamiento del consumidor y usuario Generación Millennial, desarrollada por expertos en materia de mercadotecnia turística y gastronómica, en las cuales se apoyó este proceso de indagación, son convergentes con los hallazgos obtenidos en la aplicación del sondeo flash online. Es indudable que los resultados coinciden satisfactoriamente con las aportaciones antes mencionadas, como es el hecho que estas personas se distinguen por ser un segmento de mercado atractivo para las empresas turísticas que se encuentran en posición de vanguardia en el sector de hospedaje o alojamiento considerando que ellos llevan a cabo el proceso de reservación a través de medios electrónicos, principalmente el uso de la computadora y el teléfono celular. Otra característica es que lo planean de manera anticipada con un lapso 
GENERACIÓN MILLENNIAL: CONSUMO GASTRONÓMICO Y TURÍSTICO

aproximado de uno a seis meses, y específicamente los Millennials mexicanos viajan considerablemente a sitios nacionales y muy poco a los internacionales y tienen una fuerte predilección por la comida personalizada.

En lo que corresponde al aspecto gastronómico, se reporta que tienen un consumo de alimentos fuera de casa notable de tres veces por semana, y lo hacen principalmente por placer, seguido por las condiciones de trabajo.

Para las empresas que quieren tener una importante participación en este segmento de mercado que se caracteriza por ser exigentes y conocedores a detalle de las opciones de consumo tanto turístico como gastronómico que se ofertan, necesitan involucrarse de cerca con los Millennials y proporcionarles los productos y servicios de calidad de acuerdo a sus expectativas y de esta manera lograrán que permanezcan constantemente satisfechos, sobre todo por lo que ellos representan, esto toma importancia relevante, ya que serán un porcentaje muy grande de fuerza laboral a nivel global para los próximos años.

\section{REFERENCIAS BIBLIOGRÁFICAS}

Barragán. (2015). 10 cosas que aman los millennials (y 8 que odian). Revista Merca2.0. Recuperado de: https://www.merca20.com/10-cosas-aman-millennials-8-odian/.

Burkhard, Kow y Fuggle. (2016). Informe tendencias de turismo 2017, TrekkSoft ebook library. Recuperado de: https://www.trekksoft.com/hubfs/ES_Trends_2017.pdf.

Camacho Domínguez, M. y Camacho Gómez, M. (2017). El valor del alimento en el turismo gastronómico. Revista Ideas CONCYTEG, p. 162.

Consumolab un centro AINIA (2015). Tendencias del sabor. Recuperado de: http://www.ainia.es/documentos/ebook-tendencias- sabor/.

Deloitte (2014). Resumen ejecutivo Enero "Big demands and high expectations the deloitte Millennial survey". Recuperado de: https://www2.deloitte.com/content/dam/Deloitte/global/Documents/About-Deloitte/gx-dttl2014-millennial-survey-report.pdf.

Diccionario de la lengua española (2017). Real Academia Española, Edición del Tricentenario. Recuperado de: http://dle.rae.es/?w=diccionario 
GENERACIÓN MILLENNIAL: CONSUMO GASTRONÓMICO Y TURÍSTICO

Gutiérrez-Rubí, A. (2014). 6 rasgos clave de los Millennials, los nuevos consumidores. Revista Forbes México. Recuperado de: https://www.forbes.com.mx/6-rasgos-clave-de-losmillennials-los-nuevos-consumidores/.

Hundredrooms y la escuela de negocios ESERP. (2017). Hábitos turísticos: ¿cómo viajan los millennials? Recuperado de: https://es.eserp.com/wp-content/uploads/2017/01/InformeMillenials-HR-ESERP.PDF.

Kotler, P., García de Madariaga J., Flores J., Bowen J. T. \& Makens J. C. (2011). Marketing turístico (5ª ed). Madrid: Pearson Educación, S. A.

Larousse Cocina. (2017). Diccionario de Larousse cocina. Recuperado de: https://www.laroussecocina.mx/diccionario/definicion/gastronomi.

McCrindle, M. (2014). The $A B C$ of $X Y Z$; Understanding the global generations. Australia: McCrindle.

Mesa editorial Merca2.0 (2015). 3 Factores que benefician tu estrategia de marketing gastronómico. Revista Meca2.0. Recuperado de: https://www.merca20.com/3-factoresque-benefician-tu-estrategia-de-marketing-gastronomico/.

Münch, Sandoval, Torres y Ricalde. (2015). Nuevos fundamentos de mercadotecnia. Hacia el liderazgo del mercado. México: Trillas.

Nielsen (2015). Reporte de estudio global "Estilos de vida generacionales." Recuperado de: https://www.nielsen.com/content/dam/nielsenglobal/latam/docs/reports/2016/EstilosdeVida Generacionales.pdf.

Ojeda, C.D. \& Mármol, P. (2016). Marketing turístico. Madrid: Paraninfo.

PwC, University of Southern California \& la London Business School (2013). PwC's NextGen: A global generational study (PDF). Recuperado de: http://www.pwc.com/gx/en/hrmanagement-services/pdf/pwc-nextgen-study-2013.pdf.

Schiffman y Lazar. (2010). Comportamiento del consumidor. México: Pearson Educación.

Synchrony Financial. (2014). Balancing Multi-Generational Retail Strategies Winning over Millennials without losing Boomers. Recuperado de: https://www.synchronyfinancial.com/8131_SYN_MultiGenWP.pdf. 